\title{
Introduction
}

Getting our social work or health care practice 'right' is crucial. Yet what constitutes good social work or health care is often a matter for contention. This book, The Critical Practitioner in Social Work and Health Care, sheds light on what underpins good practice. It is based on the understanding that practice in social work and health care is always problematic in some way. The practitioner always has to deal with changing demands and expectations and thus must be open to new knowledge and perspectives. The critical practitioner must then interrogate and analyse these new situations to arrive at 'best practice'. There is no final stock of knowledge that can permanently equip social work and health care professionals. Their job involves addressing uncertainty and being open to change.

We believe that the central quality and approach that a practitioner must have in addressing uncertainty is the possession of a critical stance. By critical we do not mean being negative or pessimistic. We do mean that the practitioner needs to be sceptical and evaluative about their own practice and of practice situations. This includes the policy and procedures that form the context for their practice. And this is not navel-gazing. A questioning approach to practice is essential if we are to avoid resource-led, rule-driven practice which pays little attention to what service-users want or need. While policies and procedures help guide our actions to what, we hope, generally 'works', practitioners have to be able to 'think outside the box'.

Uncertainty and a 'critical approach' may be mistakenly associated with an inability to focus on what is practical and needed. It may be incorrectly linked with a certain lack of personal and professional confidence. A distinction can be made between different types of confidence. On the one hand 'false confidence' in which a practitioner is defensive and bases their actions only on a bureaucratic rule - almost denying their professional autonomy and discretion. On the other hand 'real confidence' allows the practitioner to justify their actions based on their critical scrutiny of situations, on available evidence and a clear understanding of their role in the situation. This book is aimed at equipping practitioners to think about, to analyse and reflect upon their own practice and that of their employing agency, to enable them to justify their actions rather than being merely defensive of them. We hope readers will find that this book is a powerful tool in coming to terms with the various uncertainties they may face. Yet uncertainty means that this book cannot be exhaustive or comprehensive. What we can do is to deal with some of the major dimensions or challenges that practitioners confront.

This book should be useful to a range of professionals in the social work, social care and health care fields working in the UK and some related jurisdictions. Readers need to be aware, however, that it is also a Course Reader for an Open University course called K315 Critical Social Work Practice. This course is part of the Open University's Social Work Degree Programme and the book reflects some of the needs of this course. It should also be noted that the book is based on a previous publication, Critical Practice in Health and Social Care (Brechin, Brown and Eby, 2000). Some of the chapters in the current edition are updated and revised versions of those contained in the previous publication. 


\section{The structure of this book}

In Chapter 1 Ann Glaister introduces us to the idea that critical practice is an interdisciplinary concept. Following other authors she defines the components of an individual's critical practice as critical action, critical reflexivity and critical analysis. However, we learn that this can never be done in an isolated way, as critical practice involves constant discussion with peers and service-users to find the right approach to a given problem or issue. Critical practice must also be supported by a range of principles, firstly that of 'respecting others as equals', and secondly that practitioners must always be open to new knowledge, allowing challenge to their preconceptions. This also means being aware that sometimes the practitioner does not have knowledge appropriate to a given situation. Defining what we need to learn is a hallmark of good practice and not an admission of professional failure. However, it would be a professional failure were we not to act to obtain appropriate knowledge when our lack of knowledge becomes clear. Glaister also links critical practice with developments in critical theory and social constructionism. The author suggests that the focus of critical practice is ultimately on 'making a difference' by forging relationships to empower others; critical practice inherently involves addressing and opposing oppression and discrimination.

In Chapter 2 Mike Burt and Aidan Worsley consider professionalism in the context of the continued rise of regulatory frameworks. Using a sociological perspective, the authors debate what a profession is. They critique both the process and the traits of a profession and argue that the concept is complex and hard to pin down. Using some of the broad themes from this analysis the authors go on to relate them specifically to social work. In particular they do so in the context of what they see as the shift from specific therapeutic/ intervention roles to broader roles. They question whether the growth of so-called bureaucratic or statutory social work actually distances the social worker from the community, and then consider not just how social work draws boundaries around itself, but how it differentiates itself from others. Today's social workers are expected to relate to other professionals and to work well in complex settings. The authors discuss whether the complementary or conflicting opportunities for social workers to embrace the complexity of peoples' relationships with other professions are at odds with the focus on service-users' relationships with others.

The remainder of the chapter identifies six main areas which explore the connection between regulatory frameworks and social work as a profession. The first area, registration and protection of title, considers the historical development of the call for registration and asks whether this strengthens social work as a profession. Other areas covered include the impact of codes of practice and the role of the different Care Councils, the value of the occupational role, the impact of the social work degree and, lastly, the various nationspecific reviews of social work. The authors conclude that the contested nature of social work is such that there will always be threats and opportunities. They draw together what they see as the pertinent issues in this debate and urge that as a profession social work has to be proactive.

In Chapter 3 Hilary Brown and Sheila Barrett consider the dynamic between people who use services, carers and professionals. The chapter explores what happens when user and carer movements develop enough momentum to engage with, and challenge, the 
knowledge on which professional interventions are based. The authors consider the impact which increased service users' skills and resources for dealing with problems and creating their own networks can have. They argue that this can, in turn, affect individual relationships, as well as strategic and operational aspects within social care agencies. The chapter is divided into three sections. In the first section the authors consider the theory generated by user groups and its practical implications, and offer a case example. In the second section they critique how feedback from people who use services and carers is listened to. Many professionals, they argue, are committed to increasing participation at an individual level, but this commitment can be compromised by resource limitations and the routine of procedural assessment designed to allocate such resources. The authors consider personcentred planning and decision making and compare this with consultation exercises. They introduce the reader to Winkler's principle of 'outside scrutiny' and that real partnership can only be based on equality. In addition, they analyse the role of complaints and, finally, discuss how services should be redesigned so that user involvement is enshrined in the decision-making structures.

Throughout Chapter 3 the authors show how complex the interaction of roles has become and argue that boundaries between people who use services, carers and professionals may become blurred. They debate the terminology used to describe the various protagonists, and point out a distinction between participation and involvement. In turn, they argue that organisations cannot 'do' participation without changing their own attitudes and structures.

In Chapter 4 Keith Edwards, Chris Hallett and Phil Sawbridge, consider the complexity of the workplace in social care and in particular the issue of how to allocate and manage workload. A key quality of the critical practitioner is to be able to anticipate the demands that may be placed on them by their managers. The ability to anticipate demands may be essential to 'surviving' in the workplace. The authors review tools for this job when they think through different ways to allocate workload. They reveal that this is not simply a technical issue - how workload is allocated is part of a culture and concerns an agency's approach to practice. Part of the complexity of workload concerns the quality of information available to practitioners and managers about service-users' needs and services. The chapter discusses the impact of Information and Computer Technologies (ICT) on how information about serviceusers is managed. The authors consider how ICT could have major beneficial effects in meeting service-users' needs; for example, posing a possible future dominated by freelance social work practitioners who engage with service-users via the Internet, more or less abandoning office-based work for some service-user groups. ICT is also seen as a key component in producing 'seamless' services that solve problems in disjointedness of service delivery. Workload and the management of information is key to 'surviving in the workplace' for the critical practitioner, but so too is an awareness of the policy context. The authors discuss 'Best Value', the nature of agency-based partnership, in the context of a general policy shift from 'professional autonomy' to 'corporate accountability'. They consider some of the underpinning knowledge needed to work with corporate complexity and multi-professional and multi-stakeholder partnerships. The chapter ends by considering some of the challenges that the critical practitioner will face or needs to anticipate from the perspectives of their managers or of the teams that he or she belongs to.

In Chapter 5, by Colin Guest and Phil Scarff, we are introduced to another important critical language; that of the basic concepts in financial accounting and budgets for practitioners in health and social care. The concepts the authors use have wide applicability 
both as tools in one's own practice and for analysing the practice of employing agencies. They consider a case study: 'Middlebrook' is a centre operated by a 'national charity', and we learn about financial issues that its manager 'Marjorie' has to tackle. They discuss Middlebrook's budget, offering us insight into accounting language that the critical practitioner can use in a variety of settings: there is explanation of 'revenue budgets', 'fixed and variable costs', 'unit costs', 'direct and indirect costs', 'overhead costs' and many other tools that improve critical practitioners' understanding of the financial basis of and for their practice.

In Chapter 6 by Barbara Prynn we change the pace and look at aspects of social work practice between 1948 and 1972. The author considers her own experience of social work in the 1960s and her research into the experiences of a number of practitioners who were social workers in the 1960s. The chapter considers the organisational context for practice both before and after the Seebohm/Kilbrandon Reforms which led to the professionalisation of social work. The author suggests that the key dimension that has changed is the nature of the relationship between the service-user and the social work practitioner. The author concludes that pressure from the political left and the political right since the early 1970s has diminished the centrality of the relationship between practitioners and serviceusers. Indeed, readers could revisit some of the previous chapters in the light of Barbara Prynn's reflections. A key issue is whether there are strengths in past practice which could contribute critically to better outcomes in the twenty-first century.

In Chapter 7, by Maureen Eby and Ann Gallagher, we are introduced to ethical practice. The authors distinguish between the concepts of 'values' and 'ethics'. Values are about what we hold dear, and concern what we believe to be the best way of acting towards others, either in our personal or professional relations. Ethics, on the other hand, concerns the systematic enquiry into the values we hold. It will note, for example, where our different values compete with one another. Ethical enquiry seeks to establish patterns of thoughts in relation to the values we hold. The chapter examines competing values in different practice contexts. It distinguishes between ethical issues and ethical problems. The authors also introduce the reader to a range of ethical approaches - approaches which underpin the development of 'ethical principles' such as are contained in 'Codes of Practice'. We also learn about the 'virtues approach', the 'duties approach', the 'consequences approach' and other moral philosophy approaches. The reader is invited to identify which approach best characterises their profession's ethical stance. Each ethical approach can become a critical analytical tool that can be used to challenge received wisdom - for example, questioning whether professional ethical codes, like the social work codes of practice, are ethically consistent or self-contradictory. Eby and Gallagher's chapter introduces us to some of the tools that practitioners need to examine critically and justify both their own and their profession's values and ethics.

In Chapter 8 Celia Keeping provides two focal points: first the understanding of research and its impact on social work practice and, second, the question of if, and how, a social work practitioner can be a researcher. The chapter is about the place of research in social work practice today. It allows the reader to explore how practitioners currently engage with research to inform their practice and to examine the question of whether or not research evidence should necessarily be the basis for all aspects of social work practice. The chapter begins by discussing the context and setting in which social workers currently operate The author critiques 'trends' such as evidence-based practice, and considers developments such as the Social Care Institute for Excellence and Making Research Count. Evidence about 
how research informs social work practice is reviewed, alongside a discussion of the nature of practitioner research and how it differs from other types of research. Consideration is given to the benefits of research in practice, including whether research offers practitioners and people who use services empowerment and protection. The chapter considers the barriers to and enablers of the use of research in practice, including what kinds of organisational culture support research in practice. The author concludes that research in practice is possible, viable and valuable for critical, analytical and reflective practice.

In Chapter 9, Linda Finlay and Claire Ballinger discuss working in teams, particularly in multi-agency settings. Teamwork, they argue, is firmly on the government's agenda, driven by the findings of various enquiries and an increasing commitment towards integrated care. Section 1 starts by exploring what constitutes co-ordinated multi-disciplinary teamwork. The authors recognise that different models of teamwork operate in practice. Section 2 challenges the assumptions behind the commonplace view that teamwork is necessarily an effective way of working, and analyses the value and limitations of teamwork. Section 3 examines the challenges to teamwork and how the conflicts inherent in multidisciplinary working can constrain attempts to collaborate. Finally, different strategies for fostering positive teamwork are explored in Section 4

In Chapter 10, by Janet Seden, we are introduced to the fact that the organisational context in which we work affects our practice. This is both obvious and subtle. We learn about how organisational structures and organisational cultures can affect our performance. Following Charles Handy's ideas we are invited to try and characterise the culture of our own organisation; is it dominated by a 'power culture', a 'role culture', a 'task culture' or a 'person culture'? We are also invited to provide an 'image' of our organisation. Such images render a thumbnail picture of what it is like to live and work within a given organisation; common images are examined - for example, organisations likened to a 'machine', an 'organism', a 'brain', a 'psychic prison'. The image helps us towards critical awareness of how far our organisation is a 'learning organisation', that is, one which is like a critical practitioner - able to learn from the challenges of change either from government directives or from the demands of service-users. Janet Seden provides critical concepts that will help practitioners to articulate how well their agency operates organisationally. She gives the example of 'appreciative inquiry' as a process by which an agency can demonstrate that it is a 'learning organisation' and by which practitioners can collect evidence of the effectiveness of agency organisation and communicate that to agency policy-makers. The chapter also addresses inter-organisational working and provides tools to analyse issues and problems in this area.

In Chapter 11, by Maureen Eby and Alun Morgan, the focus is on accountability. Practitioners are not completely free agents; they cannot perform their jobs in a context of epistemological and professional anarchy. They have discretion to act within systems of bounded rationality; they must show how their actions relate to their prescribed role, and justify to a range of stakeholders their use of discretion. Eby and Morgan provide a framework for how to understand accountability: 'social accountability', in the sense of what is generally acceptable to one's colleagues; 'ethical accountability' in being clear about the ethical approach to one's actions; 'legal accountability' in being clear about one's powers and duties under legislation; and 'professional accountability' in being clear how one's actions accord with the rights and responsibilities of a profession.

Chapter 12, by Celia Davies, examines the policy process. First she considers some of the different answers that students of public policy have given to the question 'How does 
policy get made?' Second, she explores the growing scope that new policy thinking is providing to help practitioners and others develop and share policies at local level. Davies argues that the model which assumed policy was a rational process taking place at the top of organisations and requiring tight control of implementation is being replaced by a model which recognises policy as a complex and altogether messier process with more participants, much experimentation and multiple feedback loops. Davies concludes that this is an important theme for all those who work in social work and health care and that a critical practitioner needs to be both willing and able to take part in the policy process, acknowledging the multiple perspectives that people will bring and welcoming opportunities to engage with policy development and make it relevant and supportive to practice

In Chapter 13 Barry Cooper challenges us to think critically about what continuing professional development (CPD) means. He observes that the various regulatory bodies have required post-registration training and learning (PRTL) but their aims and requirements have been quite moderate. He argues that the way we approach CPD reflects our approach to practice in general - that is, the approach of both the individual practitioner and of their employing agency. The chapter also discusses the limitations of a competency-based approach to PRTL and CPD and offers some insights for the way forward. CPD is not merely a matter of technically updating our knowledge on recent events or legislation, although that is clearly necessary. Although regulatory bodies continue to base their PRTL and CPD requirements on competency-based teaching and learning there are significant limitations to this approach after professional qualification has taken place. It may be possible to mark out competencies which prepare the practitioner for workplace uncertainties, but those very uncertainties and the diverse knowledges that follow leave elaborate competency frameworks behind. Rather, current conditions mean that meeting the challenge of obsolescent knowledge becomes the responsibility of the critical practitioner. The critical practitioner should be not simply a map-reader but a map-maker. This is an aspect of the autonomy and agency of the critical practitioner. Such an approach cannot be isolated from other current themes in practice, such as the involvement of service-users. Practitioners may construct new knowledge by being 'practitioner researchers', for example, but the crucial dynamic will be between supervisor and supervisee. Exploring new inclusive ways to practise is not merely the responsibility of the individual practitioner but, as the codes of practice recognise, it is the responsibility of employers too.

In Chapter 14 James Blewett examines the future of social work for practitioners in terms of threats and opportunities. What do the changing organisational frameworks for social work practice mean for practitioners? Will social work survive as a professional discipline? Blewett reviews social policy developments leading up to the period of the New Labour governments. He then analyses how New Labour thinking has affected social work in relation to adult services, and to children and family services. He argues that there is a threat, arising from developments in social policy, that professional social work will face a diminished role and increasing marginalisation. Social work has already developed a culture of 'bureaucratisation and performance management', and it is likely that this will remain part of the picture for the foreseeable future. Social work will continue to face limited resources for its practice. Despite the various hazards, Blewett recognises that the picture is not simply one of an under-resourced, disempowered and stigmatised profession, strangled by bureaucracy and lacking a clear role or identity. There is a basis for optimism. He gives examples of excellent and innovative practice, in which service-users appreciate and value social workers and their services across a range of practice contexts. In a theme 
which is reminiscent of Barbara Prynn's chapter, Blewett reports that good social work is based upon the quality of the relationship with service-users. It is the relationship rather than resources that has transformational significance in social work. There is a concern that the configuration of current care services undermines the key component of relationship, but critical practitioners must find ways to promote their relationship with serviceusers as their key contribution. Nevertheless, Blewett ends his chapter with a warning that social work practitioners cannot take the existence of their profession for granted, and that critical practitioner must seek alliances with other professions but above all find ways to speak with greater clarity and confidence about their roles and tasks.

Throughout this book we challenge the reader to examine and reflect upon the world of the critical practitioner in social work and health care. Critical practice is not new. Rather, this book gives the opportunity to orientate critical practice in the past, to consider its present incarnation and to contemplate its impact as we look ahead. Critical practice is a living thing. It is at the core of all activity undertaken with service-users, carers and their communities, and ultimately reflects the skills and values which are fundamental to the professional caring role.

\section{Reference}

Brechin, A., Brown, H. and Eby, M.A. (eds) (2000) Critical Practice in Health and Social Care. London: Sage. 